\title{
Avaliação da tensão de aderência em sistema de reforço externamente aderido após exposição a protocolo de degradação acelerado
}

\author{
G.M. Dalfré ${ }^{*}$, G. A. Parsekian ${ }^{1}$, L. A. Sarti Júnior ${ }^{1}$, C. J. R. V. Araújo ${ }^{2}$ \\ *Autor de Contato: glaucia.dalfre@ufscar.br \\ ${ }^{1}$ Departamento de Engenharia Civil, Programa de Pós-Graduação em Engenharia Civil, Universidade Federal de \\ São Carlos, São Carlos, Brasil \\ ${ }^{2}$ Instituto de Pesquisas Tecnológicas do Estado de São Paulo, Brasil
}

\begin{abstract}
RESUMO
O presente trabalho emprega o protocolo de acondicionamento acelerado proposto pelo código americano ACI 440.9R (ACI, 2015) para avaliação da durabilidade de sistemas de reforço, sem proteção, aplicados em vigas de concreto armado. Vigas de concreto armado foram reforçadas e mantidas por 1000 horas em ambiente laboratorial ou expostas a um protocolo degradação acelerada. Posteriormente foram realizados ensaios de arrancamento para verificação da tensão de aderência entre o material de reforço e o substrato de concreto. Os resultados demonstraram que após o período de 1000 horas obteve-se um decréscimo na tensão de aderência de $5 \%$ e $39 \%$ para as vigas mantidas em ambiente laboratorial ou expostas a degradação acelerada, respectivamente, quando os dados obtidos são comparados aos das amostras de referência.
\end{abstract}

Palavras-chave: sistemas de reforço, concreto, CFRP, durabilidade, degradação acelerada.

\section{INTRODUÇÃO}

A utilização dos polímeros reforçados, ou estruturados, com fibras (FRP, Fiber Reinforced Polymer, em língua inglesa) se justifica em aplicações de reforço estrutural devido às vantagens como elevada resistência à corrosão, redução do peso da estrutura reforçada, baixa densidade, alta resistência à tração e para alguns casos, como vistos nas fibras de carbono, e elevado módulo de elasticidade.

Apesar das inúmeras vantagens e crescente implantação de sistemas de reforço em FRP aplicados por meio da técnica EBR, a ampliação do estudo torna-se indispensável devido ao contato direto e exposição do material com o ambiente externo, podendo tornar o sistema vulnerável quando submetidos às condições ambientais agressivas tais como locais com elevado teor de umidade, altas temperaturas, desgastes mecânicos e ambientes com alta concentração de agentes químicos. Para além disso, soma-se a escassez de trabalhos, com produtos disponíveis no mercado nacional, que abordem o tema durabilidade de sistemas de reforço com FRP.

Considerando a variabilidade de condições em que o sistema de reforço, juntamente com o elemento de concreto armado, pode estar exposto, o presente estudo tem por finalidade avaliar o comportamento de sistemas de reforço EBR CFRP submetidos a um ambiente com alta umidade e temperatura controlada, analisando a possível ocorrência de degradação que este meio pode ocasionar o longo do tempo. 
O estudo da verificação da degradação de um determinado material compósito pode durar anos pois tal processo está diretamente ligado ao ambiente degradante onde estão inseridos. Assim, dependendo do ambiente de exposição, as propriedades mecânicas dos materiais compósitos podem ser afetadas de forma amena (quando expostos a ambientes internos e protegidos) ou de forma mais severa (quando expostos a ambientes externos e meios agressivos), podendo resultar em alterações significativas em seu comportamento.

Silva (2014) observou que a utilização de ensaios acelerados é uma alternativa viável para verificação da durabilidade de materiais compósitos nos casos em que a falha está associada a degradação do material. Portanto, quando a degradação de um determinado material ou elemento é muito lenta ou desconhecida, incorporam-se variáveis (constantes ou alternadas, tais como temperatura elevada, umidade, voltagem, intemperismo, pressão, reagentes químicos, dentre outras), com o intuíto de acelerar o processo e, consequentemente, induzir sua falha, levando a um conhecimento mais profundo sobre o comportamento do objeto de estudo (BRUNO, 2018).

\subsection{Protocolo de degradação acelerada - ACI 440.9R (ACI, 2015)}

O código americano ACI 440.9R (ACI, 2015), utilizado nesta pesquisa, recomenda o uso de ACPs (Accelerated Conditioning Protocols, na língua inglesa) para a avaliação da durabilidade de materiais compósitos. Os ambientes de exposição recomendados são:

- Standart laboratory conditions: ambiente laboratorial de referência, o qual é caracterizado pelo uso de temperatura e umidade relativa de $23{ }^{\circ} \mathrm{C} \pm 3{ }^{\circ} \mathrm{C}$ e $50 \% \pm 10 \%$, respectivamente;

- Continuous immersion in water: imersão contínua em água potável com temperatura controlada de $50{ }^{\circ} \mathrm{C} \pm 3{ }^{\circ} \mathrm{C}$;

- Continuous exposure to humidity: exposição contínua a umidade de $100 \%$ com temperatura controlada de $60{ }^{\circ} \mathrm{C} \pm 3{ }^{\circ} \mathrm{C}$; e

- Continuous immersion in alkaline solution: imersão contínua em solução alcalina com temperatura controlada de $50{ }^{\circ} \mathrm{C} \pm 3{ }^{\circ} \mathrm{C}$.

Para tal, o código ACI 440.9R (ACI, 2015) recomenda no caso de uso de sistemas de reforço, que sua aplicação seja feita seguindo as diretrizes propostas pelo código ACI 440.2R (ACI, 2017). Assim, após o período de cura, o elemento reforçado deve ser inserido em um tanque, com geometria definida de modo a atender a demanda de ensaios a serem realizados, com a presença de água potável, solução alcalina ou umidade constante, de acordo com o método de aceleração da degradação selecionado. A Figura 1 apresenta o esquema de ensaio proposto pelo código americano ACI 440.9R (ACI, 2015) para realização do protocolo de acondicionamento.

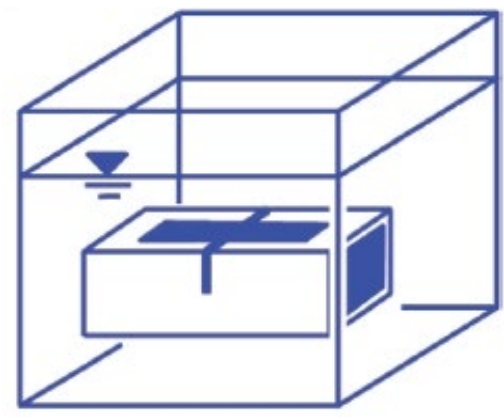

Figura 1. Arranjo de ensaio proposto para exposição à degradação acelerada.

Fonte: Adaptado de ACI 440.9R (ACI, 2015)

De acordo com o protocolo ACP, a temperatura da água potável ou solução alcalina deve ser mantida ente $50{ }^{\circ} \mathrm{C} \pm 3{ }^{\circ} \mathrm{C}$. Porém, não é definida pelo código ACI 440.9R (ACI, 2015) a forma de 
aquecimento do líquido. Assim, o pesquisador deve escolher a fonte de aquecimento e o mecanismo de controle da temperatura que melhor atenda os objetivos de sua pesquisa.

O período de duração dos ACPs pode ser definido igual a 1000 ou 10000 horas. Porém, um período intermediário de 3000 horas de exposição é proposto pelo código ACI 440.9R (ACI, 2015). Assim, testes com períodos diferentes do proposto pelo código podem ser realizados, ficando a cargo do pesquisador a sua definição.

Os ensaios mecânicos para verificação do comportamento do elemento reforçado pósacondicionamento devem ser conduzidos, após o término do período de exposição, dentro de um intervalo de 2 a 5 horas. Recomenda-se, também, a realização de ensaios do tipo "pull off" para avaliação da aderência entre o sistema de reforço e o substrato de concreto. Tais ensaios podem realizados em regiões estratégicas nas faces do elemento reforçado de acordo com a norma ASTM D7522 (ASTM, 2015) onde, por meio do arrancamento de pastilhas cilíndricas aplicadas nas regiões livres de interferências, obtêm-se a tensão de aderência. A Figura 2 ilustra o procedimento de ensaio de aderência, o qual será utilizado no pesente trabalho.

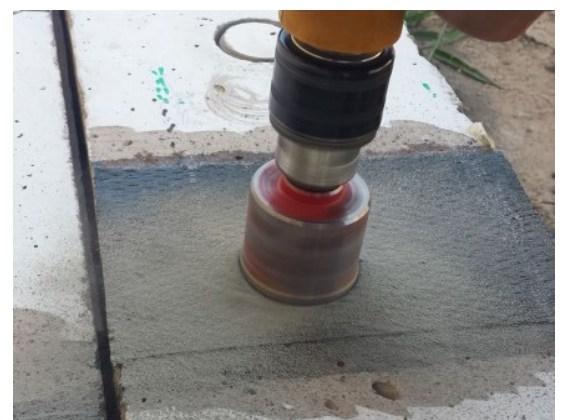

(a)

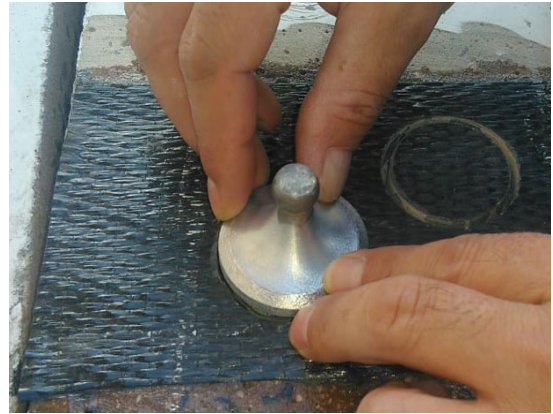

(b)

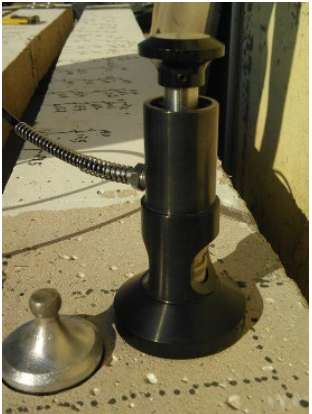

(c)

Figura 2. Ensaio de aderência: (a) execução do corte da área de ensaio, (b) colagem das pastilhas e (c) aparato posicionado sobre a pastilha.

Fonte: Adaptado de Ferreira (2019)

\section{METODOLOGIA}

O comportamento de elementos de concreto armado reforçados com FRPs é influenciado por uma série de fatores tais como: a geometria do elemento reforçado, a resistência à compressão do concreto, a taxa de armadura, a taxa e o tipo de fibras utilizadas no compósito de FRP, a matriz polimérica utilizada, a configuração do sistema de reforço, a técnica de reforço utilizada e o ambiente ao qual a estrutura está exposta.

Nesse sentido, este trabalho buscou avaliar o comportamento do sistema de reforço com mantas de CFRP aplicadas segundo a técnica EBR quando exposto a degradação acelerada de acordo com o protocolo ACP, proposto pelo código americano ACI 440.9R (ACI, 2015), cujo método de aceleração de degradação escolhido é baseado na exposição de vigas de concreto armado, sem reforço e reforçadas, à umidade constante com temperatura controlada de $50{ }^{\circ} \mathrm{C} \pm 3{ }^{\circ} \mathrm{C}$.

Para isso, vigas de concreto armada reforçadas segundo a técnica EBR e os materiais intervenientes (resinas epoxídicas e compósitos de CFRP) foram acondicionados em dois locais distintos:

- Ambiente laboratorial (interno e protegido), e

- Ambiente com umidade constante obtida por meio de imersão do sistema de reforço em água potável, obtida da rede pública de abastecimento, com temperatura controlada, seguindo as recomendações do código ACI 440.9R (ACI, 2015).

O principal aspecto analisado neste trabalho foi o incremento ou decréscimo da tensão de aderência entre o sistema de reforço e o substrato de concreto das vigas reforçadas após exposição aos ambientes anteriormente apresentados nas idades de 14 e 87 dias (1000h). 
O programa experimental é composto por vinte e oito vigas biapoiadas com dimensões de $120 \mathrm{~mm}$ $\times 200 \mathrm{~mm} \times 250 \mathrm{~mm}$, tal como apresentado na Figura 3, as quais possuem concreto com resistência à compressão média $\left(\mathrm{f}_{\mathrm{cm}}\right)$ de $32 \mathrm{MPa}$ e armadura de flexão positiva composta por duas barras de aço CA-50 com diâmetro de $10 \mathrm{~mm}$ (taxa de armadura longitudinal de 0,75 \%). Para se evitar a ruptura por cisalhamento, estribos de aço CA-60, com diâmetro de $5 \mathrm{~mm}$ e espaçamento de $10 \mathrm{~cm}$, foram utilizados (Figura 3).

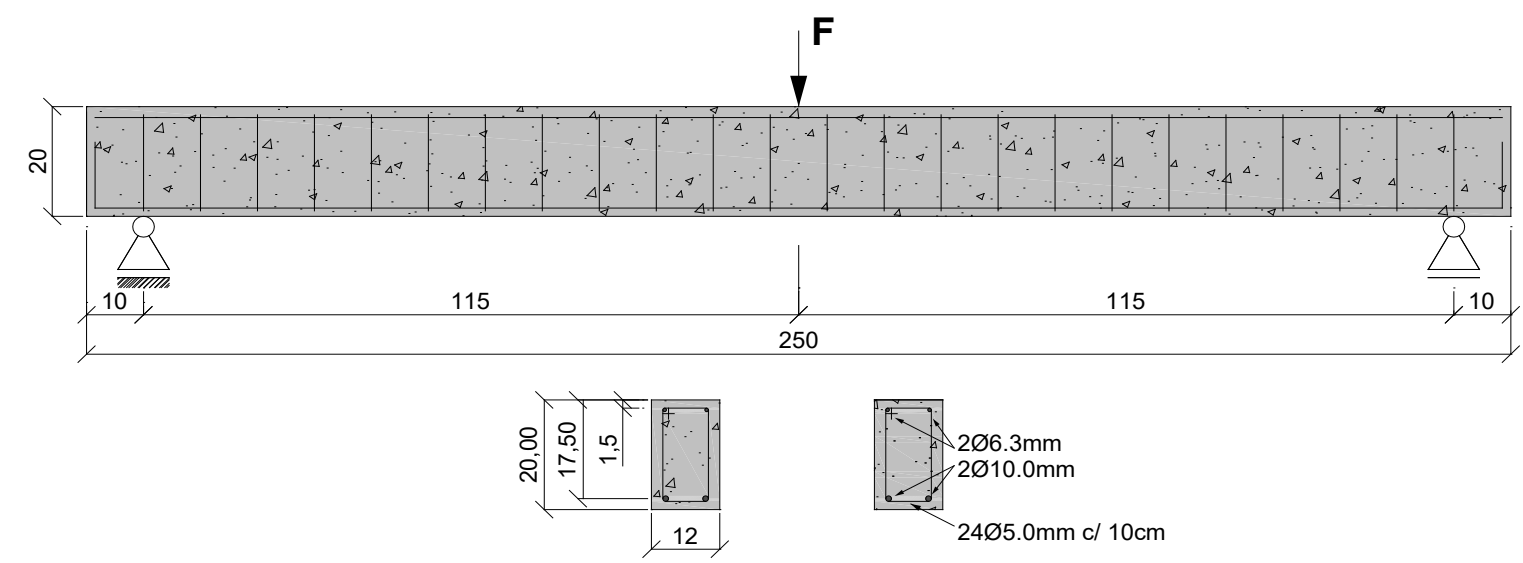

Figura 3. Característica das vigas de concreto armado. Unidades em $\mathrm{cm}$. Fonte: Ferreira (2019)

O sistema de reforço utilizado utiliza mantas de fibras de carbono aplicadas diretamente no substrato de concreto segundo a técnica EBR, nas faces inferiores das vigas (zona tracionada).

Dentre os diversos tipos de fibras disponíveis no mercado e dando continuidade aos trabalhos realizados por Ferreira (2019), optou-se por utilizar o sistema de reforço composto por mantas do tipo $C$-Sheet 240. Como matriz polimérica do sistema de reforço foram utilizados dois tipos de resinas epoxídicas: a resina $\mathrm{A}$ (primer) e a resina $\mathrm{B}$ (saturação).

Para a aplicação do sistema de reforço foi previamente realizado o tratamento da superfície de concreto, onde o mesmo seria aderido com o intuito de garantir a aderência perfeita entre o substrato de concreto e o compósito de CFRP. Para tal, realizou-se o desbaste da camada superficial, com remoção da nata de cimento e exposição dos agregados, utilizando-se um disco de desbaste diamantado acoplado a uma esmerilhadeira (Figura 4a). Na sequência, aplicou-se ar comprimido em toda a superfície para limpeza e remoção das partículas sólidas uma vez que é fundamental que a superfície de concreto esteja livre de sujidades e contaminações ocasionadas pelo contato de óleos, graxas ou desmoldantes (Figura 4b).

Ao término do tratamento da superfície foi aplicado uma fina camada da resina A (primer) com o objetivo de regularizar a superfície e tamponar os poros presentes na superfície de concreto, assim, proporcionando melhor aderência entre o CFRP e o substrato de concreto. 


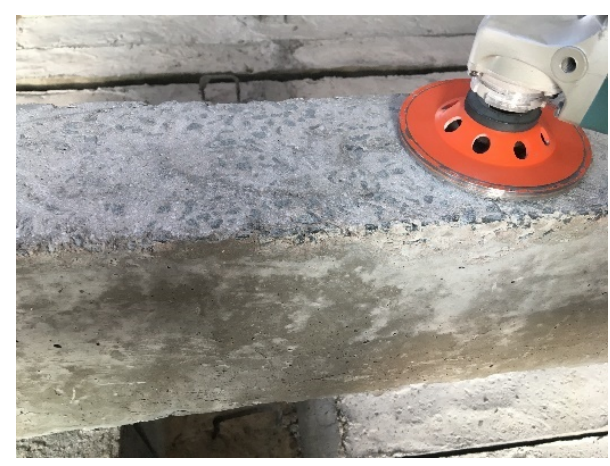

(a)

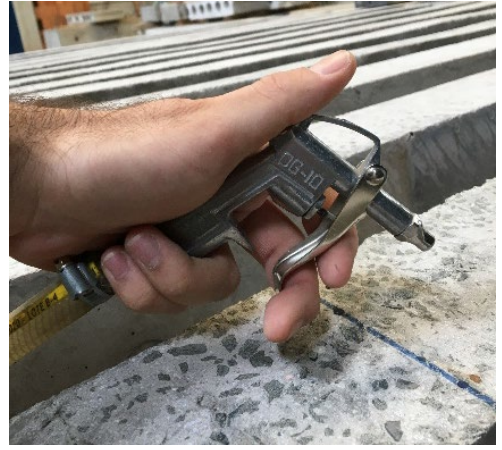

(b)

Figura 4. Preparação da superfície de concreto.

Fonte: Sarti Júnior (2020)

O posicionamento da manta no substrato de concreto iniciou-se após 40 minutos da aplicação da resina primer. Neste intervalo realizou-se a impregnação da manta, previamente cortada e limpa, com a resina B. Em seguida procedeu-se o posicionamento e colagem das mantas de fibra de carbono, utilizando-se também a resina de saturação. Após o posicionamento e alinhamento da manta na superfície, um rolo de borracha foi utilizado para remover o excesso de resinas e possíveis bolhas que possam ter sido formadas no tardoz da manta. O preparo das resinas primer e saturação seguiu as recomendações do fabricante, o qual indica a agitação dos produtos, pesagem dos componentes A e B na proporção de 2 partes do componente A (monômero) para 1 parte do componente B (catalisador), seguido da mistura mecânica, por um período de 3 minutos, realizada com o uso de um misturador metálico acoplado a uma furadeira.

De acordo com o fabricante, para que aplicação do sistema de reforço possa ser realizada, a temperatura da superfície de concreto deve apresentar temperaturas de, no mínimo, $8^{\circ} \mathrm{C}$, e também deve estar $3^{\circ} \mathrm{C}$ acima da temperatura do ponto de orvalho. Neste sentido, no ato da aplicação do sistema de reforço, a umidade ambiente de $48 \%$ e as temperaturas ambiente, do substrato de concreto e do ponto de orvalho de $26^{\circ} \mathrm{C}, 21^{\circ} \mathrm{C}$ e $15,4^{\circ} \mathrm{C}$, respectivamente, foram aferidas.

Para avaliar o comportamento e durabilidade das vigas, dois ambientes de exposição foram adotados nesta pesquisa, os quais são apresentados a seguir:

- Ambiente laboratorial (LAB): interno, protegido, o qual serviu como referência aos demais ensaios (Figura 5a-b). O ambiente adotado como laboratorial é o Laboratório NETPRE (Núcleo de Estudo e Tecnologia em Pré-Moldados de Concreto) da Universidade Federal de São Carlos (UFSCar), o qual é coberto e protegido de intempéries, e

- Tanques de acondicionamento para avaliação da durabilidade (ACP): com imersão do sistema de reforço em água potável, obtida da rede pública de abastecimento, com temperatura constante de $50{ }^{\circ} \mathrm{C} \pm 3{ }^{\circ} \mathrm{C}$, Figura 5(c-f).

Tendo em vista tais ambientes, a designação utilizada para identificação de cada viga foi Vx_y_w$z$, onde " $\mathrm{x}$ " é o número do elemento ensaiado ( 1 ou 2), " $y$ " corresponde aos elementos mantidos em ambiente laboratorial (LAB, de Laboratory) ou expostos ao protocolo de acondicionamento acelerado (ACP, de Acelerated Conditioning Protocol), "w" devido a utilização ou não de material de reforço (0 ou CFRP) e " $z$ " corresponde a data de realização do ensaio (14 dias ou 1000 horas (87 dias) após a aplicação do sistema de reforço). Um resumo do programa experimental é apresentado na Tabela 1. Note-se que as vigas foram posicionadas no tanque de degradação quando o sistema de reforço atingiu a idade de 14 dias. 


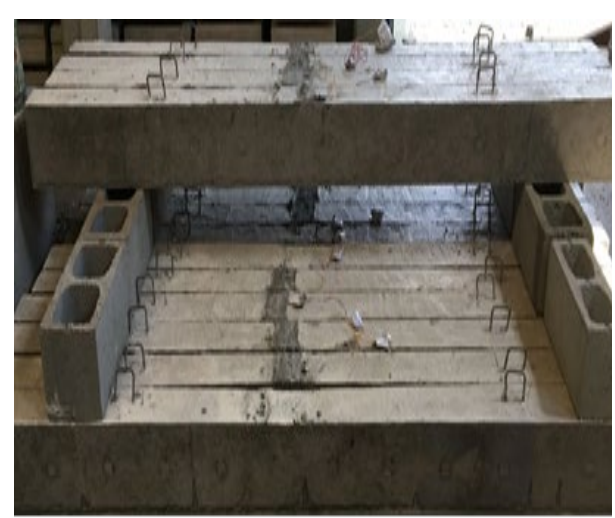

(a)

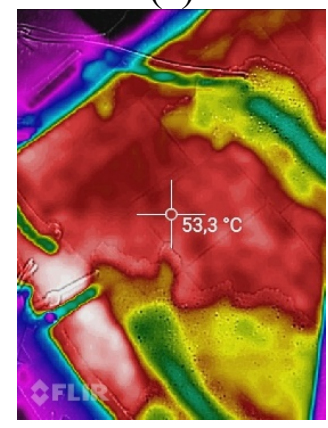

(d)

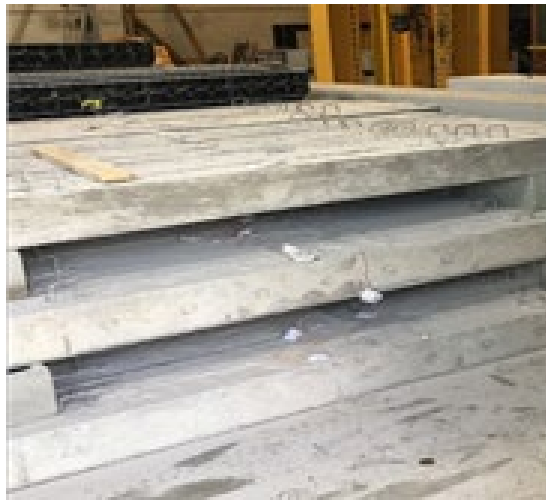

(b)

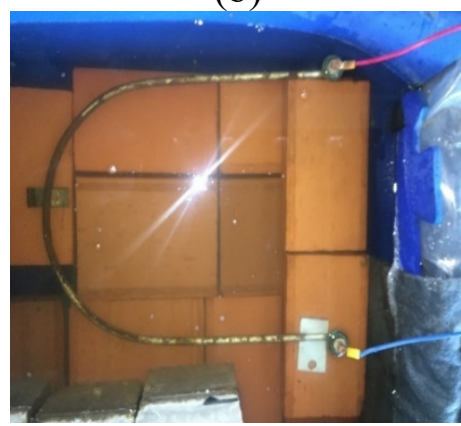

(e)

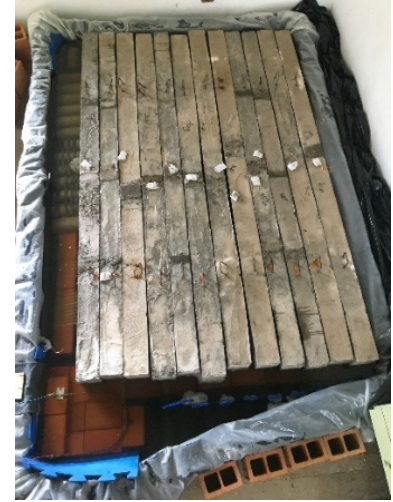

(c)

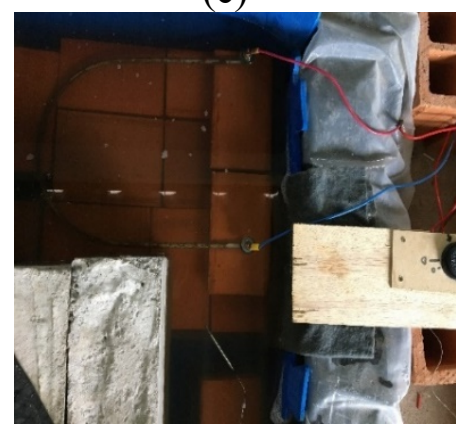

(f)

Figura 5. Locais de execução da campanha experimental de exposição dos elementos a degradação acelerada: (a-b) ambiente laboratorial, (c) tanque de umidade constante, (d) imagem termográfica, (e) resistência elétrica e (f) termostato e resistencia.

Fonte: Sarti Júnior (2020)

Tabela 1. Resumo do programa experimental

\begin{tabular}{|c|c|c|c|}
\hline Idade do ensaio & Ambiente de exposição & Identificação & Total \\
\hline \multirow{2}{*}{$\begin{array}{l}45 \text { dias (ou } 14 \text { dias } \\
\text { após aplicação do } \\
\text { sistema de reforço) }\end{array}$} & \multirow{2}{*}{$\begin{array}{l}\text { Ambiente laboratorial } \\
\text { (Referência) }\end{array}$} & $\begin{array}{l}\text { V1_LAB_0_14d } \\
\text { V2_LAB_0_14d }\end{array}$ & 2 \\
\hline & & $\begin{array}{l}\text { V1_LAB_CFRP_14d } \\
\text { V2_LAB_CFRP_14d }\end{array}$ & 2 \\
\hline \multirow{4}{*}{$\begin{array}{l}1000 \text { horas ( } 87 \text { dias) } \\
\text { após aplicação do } \\
\text { sistema de reforço }\end{array}$} & \multirow{2}{*}{ Ambiente laboratorial } & $\begin{array}{l}\text { V1_LAB_0_1000h } \\
\text { V2_LAB_0_1000h }\end{array}$ & 2 \\
\hline & & $\begin{array}{l}\text { V1_LAB_CFRP_1000h } \\
\text { V2_LAB_CFRP_1000h }\end{array}$ & 2 \\
\hline & \multirow{2}{*}{$\begin{array}{l}\text { Tanques de } \\
\text { acondicionamento com } \\
\text { temperatura controlada }\end{array}$} & $\begin{array}{l}\text { V1_ACP_0_1000h } \\
\text { V2_ACP_0_1000h }\end{array}$ & 2 \\
\hline & & $\begin{array}{l}\text { V1_ACP_CFRP_1000h } \\
\text { V2_ACP_CFRP_1000h }\end{array}$ & 2 \\
\hline
\end{tabular}

Fonte: Sarti Júnior (2020)

Atualmente, utilizam-se os ensaios de aderência, conhecidos como pull-off como forma de controle tecnológico nas obras de engenharia civil. Neste sentido, a tensão de aderência no substrato de concreto das vigas de concreto armado e a aderência entre o compósito de CFRP com o substrato de concreto foram avaliadas no presente trabalho. Os ensaios de pull-off foram conduzidos após 14 dias de cura do sistema de reforço e, também, após o período de 1000 horas. 
O código americano ACI 440.9R (ACI, 2015) recomenda que o ensaio seja realizado nas extremidades da viga de concreto armado. Entretanto, devido à geometria da viga de concreto armado, não foi possível atender a tal recomendação devido a presença dos ganchos, da armadura de ancoragem, nesta região, o que poderiam interferir nos resultados obtidos. Neste sentido, os ensaios foram conduzidos nas faces laterais das vigas de concreto armado, em uma região onde não havia presença de armadura e sem padrão fissuratório visível após a realização dos ensaios mecânicos de flexão (Figura 6). Apresenta-se na Figura 7 o processo de aplicação do sistema de reforço para os ensaios de aderência.

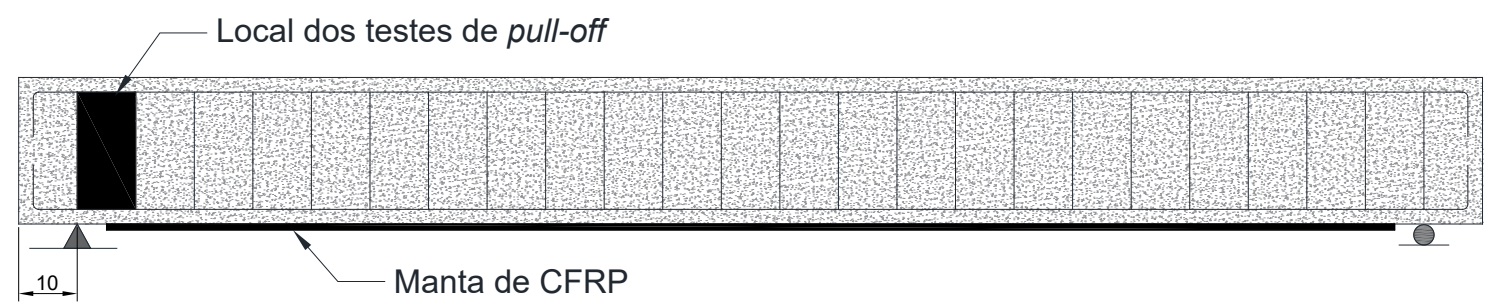

Figura 6. Região de aplicação do CFRP nas vigas de concreto armado reforçadas. Fonte: Sarti Júnior (2020)

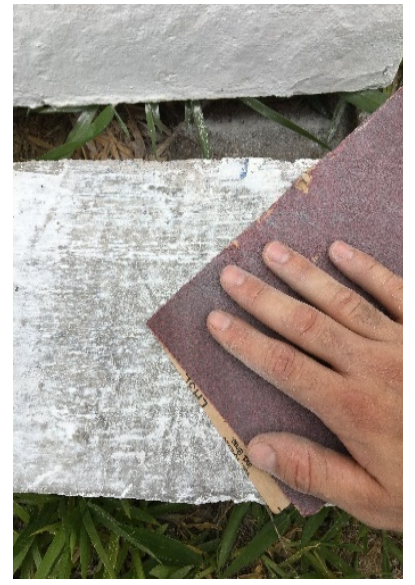

(a)

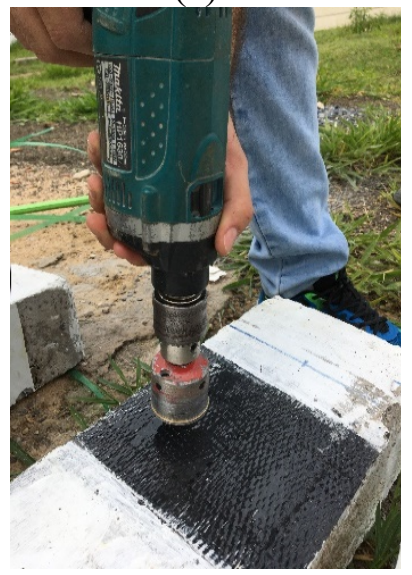

(e)

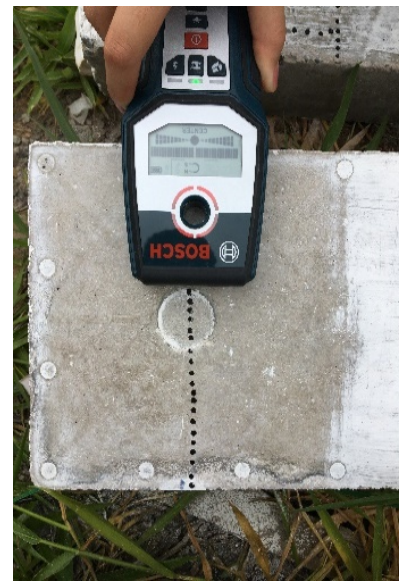

(b)

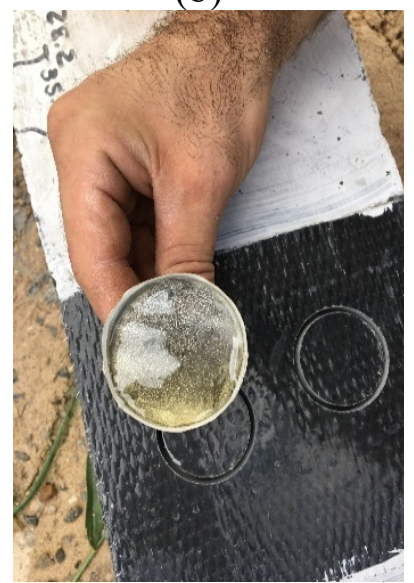

(f)

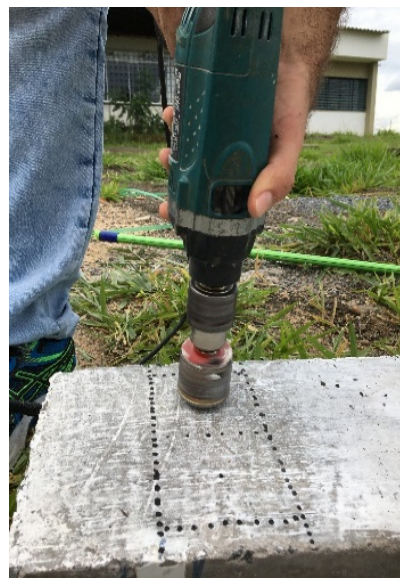

(c)

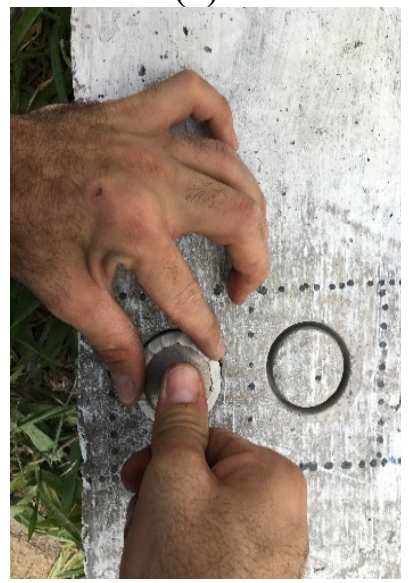

(g)

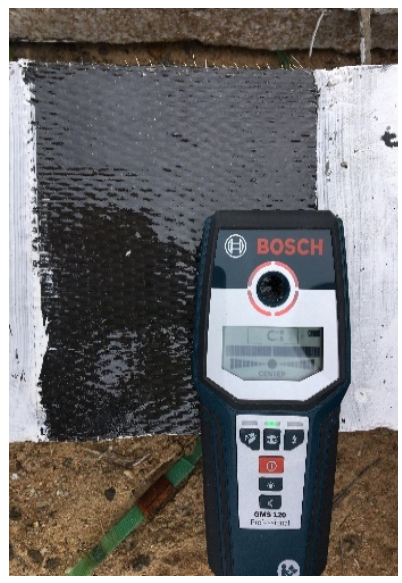

(d)

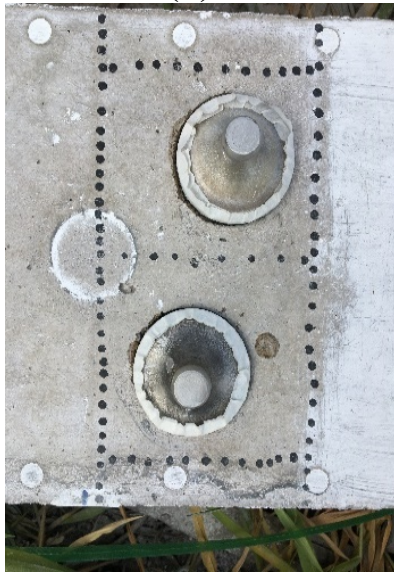

(h)

Figura 7. Procedimento de aplicação das pastilhas: (a) limpeza da superfície da viga; (b) pacometria na região pré-definida; (c) cortes nas áreas demarcadas; (d) pacometria sobre o sistema de reforço; (e) cortes sobre o CFRP; (f) aplicação de resina na pastilha; (g) aplicação da pastilha e (h) aspecto final das pastilhas aplicadas.

Fonte: Sarti Júnior (2020) 
O ensaio foi precedido da pacometria da viga com o intuito de conhecer o real posicionamento da armadura e, posteriormente, pela definição da área sem quaisquer tipos de interferência. Após a delimitação da região de ensaio fez-se o tratamento da superfície com o auxílio de uma lixa, a qual removeu a camada de tinta anteriormente aplicada. Em seguida, aplicou-se ar comprimido para limpeza e remoção de possíveis sujidades.

Nas vigas de concreto armado reforçadas, aplicou-se uma camada do sistema de reforço, o mesmo utilizado na face tracionada, na mesma região previamente definida e tratada para as vigas sem reforço. Para tal, após a execução dos ensaios mecânicos de flexão nas vigas de concreto armado, realizaram-se cortes circulares, com uma profundidade média de $2 \mathrm{~cm}$, utilizando-se furadeira e serra copo com diâmetro de $50 \mathrm{~mm}$. Em seguida efetuou-se a colagem das pastilhas de alumínio, com diâmetro de $50 \mathrm{~mm}$, no substrato de concreto e na manta de CFRP, conforme recomendado na norma ASTM D7522 (ASTM, 2015). Apresenta-se na Figura 8 o passo a passo realizado para aplicação das pastilhas.

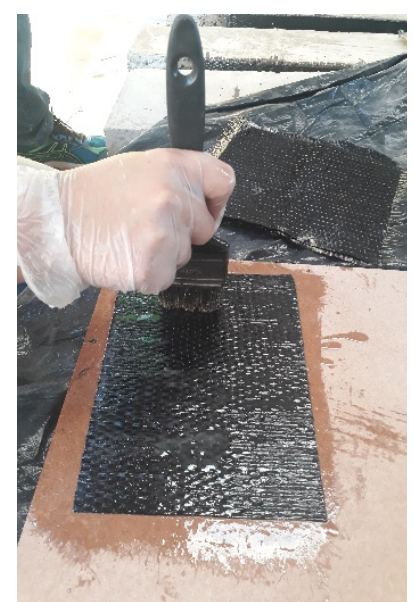

(a)

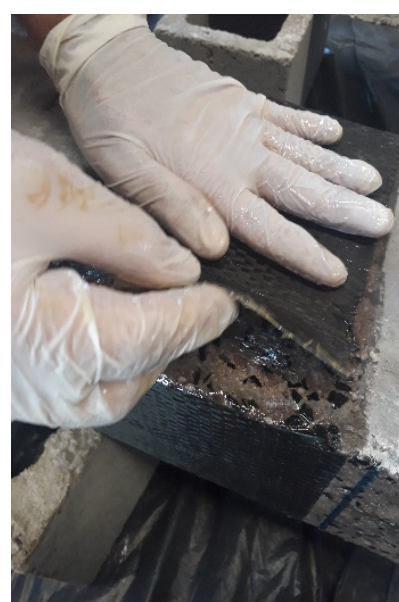

(b)

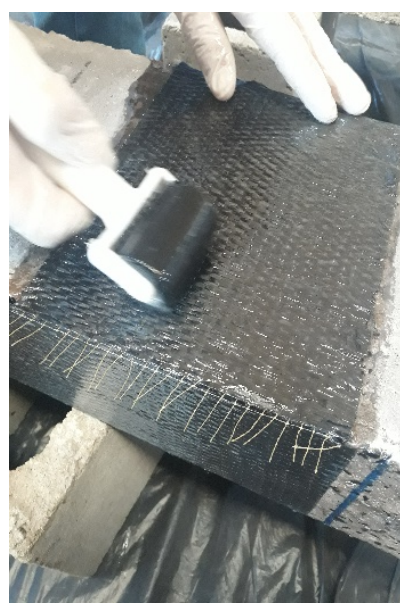

(c)

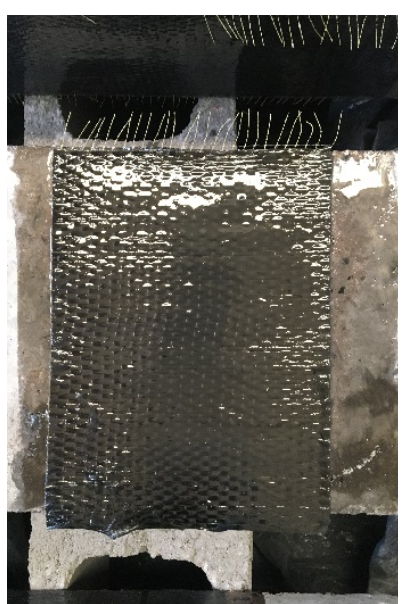

(d)

Figura 8. Processo de aplicação do CFRP para os ensaios de aderência: (a) impregnação da manta de fibra de carbono; (b) posicionamento do CFRP; (c) regularização com o rolo de borracha e (d) aparência final do CFRP aplicado.

Fonte: Sarti Júnior (2020)

Note-se que a resina epoxídica de saturação foi utilizada para a colagem das pastilhas nas áreas de ensaio e em cada viga foram coladas 2 pastilhas. $O$ período de cura seguiu os mesmos definidos para o sistema de reforço principal, 14 dias após a aplicação.

Os ensaios foram conduzidos seguindo as recomendações da norma ASTM D7522 (ASTM, 2015). Para isso, utilizou-se o equipamento de arrancamento manual da marca DeFelsko, modelo ATM50, o qual possui campo de leitura de 0,4 a 3,3 $\mathrm{MPa}$, com precisão na casa de $0,01 \mathrm{MPa}$, para a pastilhas com diâmetro de $50 \mathrm{~mm}$. A Figura 9 apresenta a metodologia aplicada na execução dos ensaios de pull-off tanto nas vigas de concreto armado sem reforço como nas vigas reforçadas. 


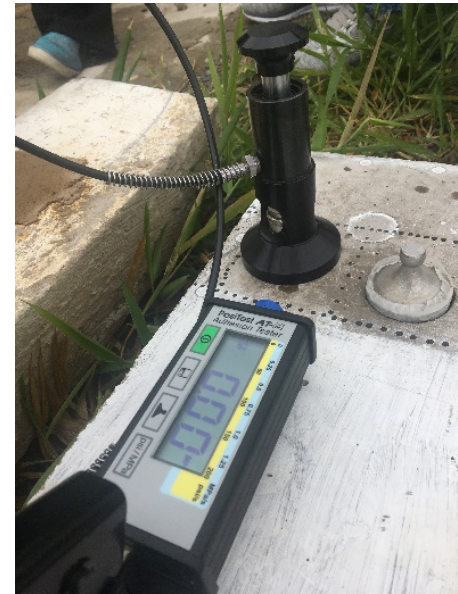

(a)

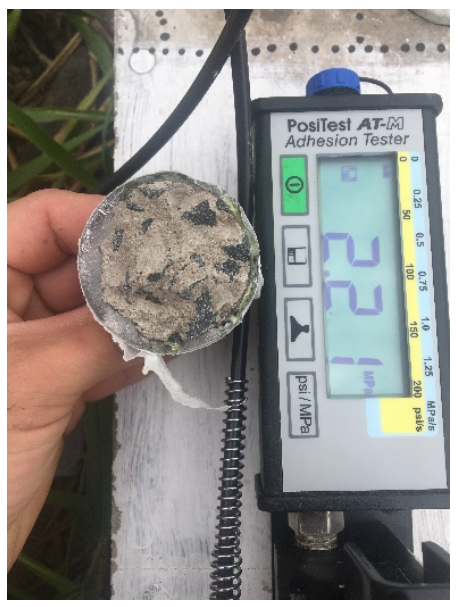

(b)

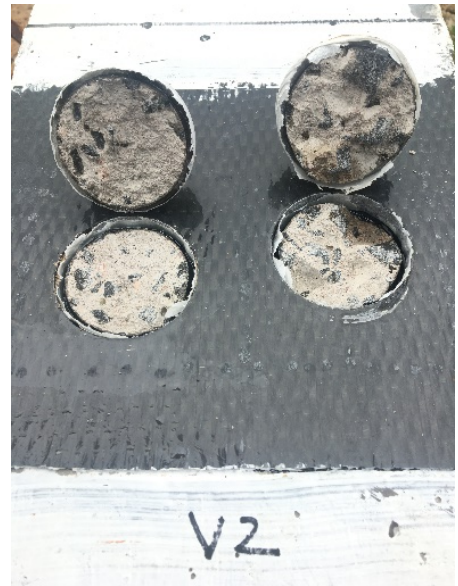

(c)

Figura 9. Metodologia utilizada no ensaio de aderência pull-off: (a) posicionamento do equipamento, (b) leitura do resultado após tração e (c) aspecto final das pastilhas após o ensaio.

Fonte: Sarti Júnior (2020)

\section{RESULTADOS}

Após a cura do adesivo estrutural utilizado (considerado como sendo 14 dias), foram conduzidos os ensaios de aderência por meio da fixação da pastilha de alumínio ao equipamento mecânico de pull-off, e então, gradativamente, iniciou-se a aplicação da força de tração até a ruptura da pastilha colada na superfície de concreto ou CFRP.

Apresentam-se na Tabela 2 os resultados obtidos nos ensaios de aderência realizados nas vigas de concreto armado sem reforço e reforçadas. Nesta, estão contemplados os resultados obtidos nos ensaios de referência bem como os ensaios após 1000 horas de exposição aos ambientes laboratorial e protocolo de acondicionamento acelerado.

Tabela 2. Resultados médios para os ensaios de aderência realizados nas vigas de concreto armado sem reforço e reforçadas.

\begin{tabular}{|c|c|c|c|c|}
\hline $\begin{array}{l}\text { Ambiente de } \\
\text { exposição }\end{array}$ & Vigas & $\begin{array}{l}\text { Idade do ensaio } \\
\text { (dias) }\end{array}$ & $\begin{array}{l}\text { Tensão de } \\
\text { aderência } \\
\text { (MPa) }\end{array}$ & $\begin{array}{l}\text { Modo de } \\
\text { ruptura }\end{array}$ \\
\hline Referência & $\begin{array}{l}\text { V_LAB_0_14dias } \\
\text { V_LAB_CFRP_14dia } \\
\text { s }\end{array}$ & $\begin{array}{l}28 \text { após a concretagem } \\
14 \text { após a aplicação do } \\
\text { sistema de reforço }\end{array}$ & $\begin{array}{l}2,0(14,4) * \\
2,8(12,6) *\end{array}$ & $\begin{array}{l}\mathrm{S} \\
\mathrm{S}\end{array}$ \\
\hline $\begin{array}{l}\text { Laboratorial } \\
\text { (1000 horas) }\end{array}$ & $\begin{array}{c}\text { V_LAB_0_1000h } \\
\text { V_LAB_CFRP_1000h }\end{array}$ & 87 & $\begin{array}{l}2,1(2,1) * \\
2,1(5,1) *\end{array}$ & $\begin{array}{l}S \\
S\end{array}$ \\
\hline $\begin{array}{l}\text { Degradação } \\
\text { acelerada } \\
\text { (1000 horas) } \\
\end{array}$ & $\begin{array}{c}\text { V_ACP_0_1000h } \\
\text { V_ACP_CFRP_1000h }\end{array}$ & 87 & $\begin{array}{c}1,9(4,2) \\
1,7(6,1) *\end{array}$ & $\begin{array}{l}\mathrm{S} \\
\mathrm{S}\end{array}$ \\
\hline
\end{tabular}

Fonte: Sarti Júnior (2020)

Após o ensaio de aderência observou-se que algumas pastilhas não aderiram totalmente nas superfícies de colagem. Com isso, para o cálculo da tensão de aderência, utilizou-se os valores da 
área efetiva da pastilha, ou seja, considerou-se para tal somente a área aderida no substrato de concreto. Assim, com base nos fragmentos de concreto aderidos as pastilhas, foram realizados levantamentos das áreas efetivas, as quais foram fotografadas e analisados em software do tipo CAD para verificação da área de colagem efetiva. Na Figura 10 apresentam-se as imagens de algumas pastilhas pós-ensaio de pull-off e o modo de ruptura obtido.

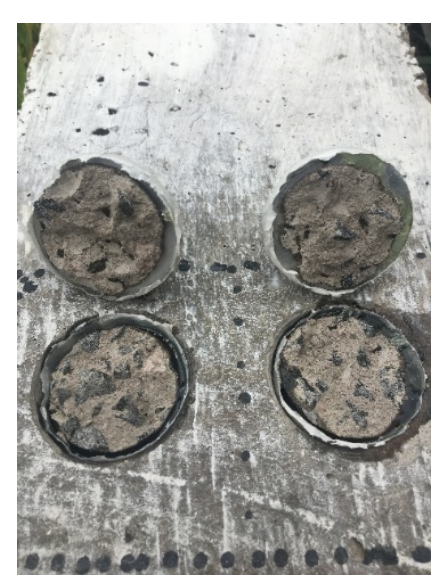

(a)

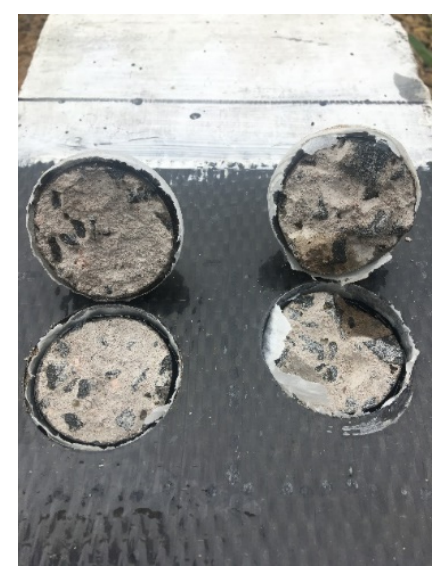

(b)

Figura 10. Pastilhas pós-ensaio de aderência pull-off: (a) viga de concreto armado e (b) sistema de reforço.

Fonte: Sarti Júnior (2020)

Com base nos resultados obtidos nos ensaios de aderência pull-off, observa-se que todas as vigas, com e sem reforço, apresentaram o mesmo modo de ruptura. Neste sentido, verificou-se que, tanto para os ensaios realizados nas vigas de referência quanto os realizados nas vigas mantidas em ambiente laboratorial e expostas ao protocolo de degradação acelerado, o modo de ruptura ocorreu no substrato de concreto e não na interface concreto/adesivo/CFRP.

Ainda que tenha sido observada uma tendência na redução da carga de pull-off, a partir da análise dos resultados e modo de ruptura verifica-se um indicativo que a aderência entre o concreto/adesivo/CFRP é superior a resistência do concreto, mesmo após exposição aos ambientes degradantes. Além disso, tais valores são superiores aos indicados na ACI 440.2R (ACI, 2015), a qual preconiza que a tensão de aderência deve ser de, pelo menos, 1,4 MPa.

\section{CONCLUSÃO}

Este trabalho apresenta um programa experimental que visa avaliar o comportamento a longo prazo de sistemas de reforço EBR CFRP. Para isso, dois ambientes de exposição foram adotados nesta pesquisa: laboratorial, o qual serviu de referência para as demais análises, e degradação acelerada do sistema de reforço baseado no protocolo de acondicionamento, visando a possível degradação dos FRPs, recomendado pelo código americano ACI 440.9R (ACI, 2015). Os resultados obtidos nos ensaios de aderência (pull-off) foram baseados na ruptura do concreto de substrato. Ainda que tenha sido observada uma tendência na redução da carga de pull-off, a partir da análise dos resultados e modo de ruptura verifica-se um indicativo que a aderência entre o concreto/adesivo/CFRP é superior a resistência do concreto, mesmo após exposição aos ambientes degradantes. 


\section{AGRADECIMENTOS}

Os autores agradecem à empresa Metrodular, de Piracicaba, SP, pelo fornecimento das formas poliméricas utilizadas na concretagem das vigas, e ao Laboratório de Polímeros do Departamento de Engenharia de Materiais (DEMa) e ao Laboratório de Sistemas Estruturais (LSE) da Universidade Federal de São Carlos (UFSCar), pela realização dos ensaios desta pesquisa. O presente trabalho foi realizado com apoio do Instituto de Pesquisas Tecnológicas do Estado de São Paulo (IPT) e da Coordenação de Aperfeiçoamento de Pessoal de Nível Superior - Brasil (CAPES) - Código de Financiamento 001.

\section{REFERÊNCIAS}

AMERICAN CONCRETE INSTITUTE. ACI COMMITTEE 440.2R. Guide for the design and construction of externally bonded FRP systems for strengthening concrete structures. American Concrete Institute, ACI Committee 440, 2017.

AMERICAN CONCRETE INSTITUTE. ACI COMMITTEE 440.9R. Guide to Accelerated Conditioning Protocols for Durability Assessment of Internal and External Fiber-Reinforced Polymer (FRP) Reinforcement. ACI Committee 440, 10p. 2015.

AMERICAN SOCIETY FOR TESTING AND MATERIALS. ASTM D7522: Standard test method for pull-off strength for FRP laminate systems bonded to concrete substrate. West Conshohocken, Pensilvânia, EUA, 2015.

BRUNO, G. U. Avaliação da degradação natural e acelerada de revestimentos orgânicos. 2018. 99 p. Dissertação (Mestrado em Engenharia na área de Ciência e Tecnologia dos Materiais) - Universidade Federal do Rio Grande do Sul, Porto Alegre, 2018.

FERREIRA, D. C. Avaliação da degradação de vigas reforçadas com FRP quando expostas ao intemperismo. 2019, 138 p. Dissertação (Mestrado em Engenharia Civil) - Universidade Federal de São Carlos, São Carlos, SP, 2019.

SARTI JUNIOR, L. A. (2020). Durabilidade de vigas de concreto armado reforçadas com mantas de CFRP submetidas a degradação acelerada. Dissertação (Mestrado). Universidade Federal de São Carlos - UFSCar. Disponível em: https://repositorio.ufscar.br/handle/ufscar/12781 (Acessado: 30 de abril de 2021).

SILVA, S. C. B. Estudo da degradação acelerada da argamassa em um sistema de revestimento cerâmico de fachadas. 2014, 129 p. Dissertação (Mestrado em Ciência e Engenharia de Materiais), Universidade do Extremo Sul Catarinense, Criciúma, SC, 2014. 